\title{
Littératures de la France médiévale
}

M. Michel ZINK, membre de l'Institut

(Académie des Inscriptions et Belles-Lettres), professeur

La question posée par le cours de l'an dernier, celle de la poésie comme récit, est si vaste, si difficile (peut-être également si confuse, en ce qu'elle oblige à combiner une définition théorique et une approche historique de la poésie), qu'il n'a guère été possible en un an que d'en analyser les termes et de n'en traiter, dans le cadre des lettres médiévales, qu'un aspect, celui de la poésie du dit. Cette année, on a tenté d'approfondir l'examen de cette question à travers l'examen d'œuvres très particulières, que nous a léguées le Moyen Âge occitan, des œuvres souvent considérées dans l'ordre littéraire avec condescendance, mais qui exercent sur leur lecteur une séduction extrême, dont il aurait tort de rougir : les récits et les commentaires en prose qui accompagnent dans certains manuscrits les chansons des troubadours. Exemple parfait, semble-t-il : des récits fondés sur des poèmes. Exemple parfait? Voire. Car des récits sur la poésie peuvent n'avoir rien de commun avec la poésie comme récit ou même avec la poésie tout court. C'est même l'opinion la plus commune s'agissant de ces récits-là. Elle n'est pas fondée. Il a fallu pour le montrer - et tel a été, dans ce cours, l'essentiel du travail - examiner de près la relation de ces récits aux poèmes qu'ils prétendent commenter, dégager l'idée, évidemment implicite, de la poésie qui les fonde et qui les guide, nous demander s'ils ne relèvent pas eux-mêmes de la poésie.

Les vidas et les razos des troubadours n'ont pour raison d'être que de préparer à l'audition de leurs poèmes, de les annoncer, de les expliquer, d'en rendre raison (razo). Ces récits tirent leur substance de poèmes. Ils n'existent que par eux. Ils entretiennent donc avec la poésie des relations plus étroites qu'aucun autre de ceux que nous a laissés le Moyen Âge. Pourtant, ils sont en prose. Tant d'autres récits brefs, au Moyen Âge, sont en vers : les lais, les dits, les fabliaux français, les novas occitanes, les romances espagnols, les Canterbury Tales de Chaucer et une infinité d'autres, composés dans toutes les langues de l'Europe. Pour ne rien dire des formes narratives longues versifiées, chansons de geste et romans. Rien de plus naturel : dans la genèse et dans la jeunesse de toutes les 
littératures, le vers apparaît d'abord, et la prose plus tard. Le critique moderne se demande quelle idée de la poésie suppose cet emploi premier du vers, et même s'il en suppose une. Mais les textes eux-mêmes ne se posent pas la question. La poésie n'appartient pas de façon visible à leurs préoccupations.

À l'inverse, les vidas et les razos des troubadours occitans sont en prose. Mais elles n'existent que par et pour la poésie. Les premières retracent la vie des poètes. Les secondes commentent les poèmes, en «rendent raison». Ces récits, comme tous les récits, ne font rien d'autre que raconter des histoires. Mais les histoires qu'ils racontent portent sur des poètes et leurs poèmes. Ces récits en prose ne prétendent en aucune façon relever de la poésie. Mais leur raison d'être est d'aider à lire la poésie. Ils se veulent tout entiers à son service. Les questions ultimes qu'ils posent, sans jamais le dire, sont celles de sa nature, de son sens et de son interprétation.

Voilà qui justifiait qu'on leur consacrât le cours d'une année dans le cadre de son thème général. Mais cette justification elle-même paraît artificielle ou artificieuse. Car, quand nous lisons les vidas et les razos, nous y découvrons avec intérêt ou avec amusement une multitude d'anecdotes inattendues, troublantes, touchantes, scabreuses ou saugrenues, mais nous ne sommes pas frappés, c'est le moins qu'on puisse dire, par leur pénétration critique dans l'ordre de la poésie. Chercher dans ces récits, qui semblent réduire à l'anecdote les chansons si peu anecdotiques des troubadours, une approche révélatrice de ce qu'est profondément leur poésie, c'est, semble-t-il, cultiver par plaisir le paradoxe et s'engager dans une quête désespérée.

Certes, l'intérêt des vidas et des razos ne se limite pas aux renseignements biographiques parfois exacts et souvent erronés qu'elles fournissent sur les troubadours. La recherche récente (Maria Luisa Meneghetti, Elizabeth Wilson Poe) a su en tirer des indications précieuses sur le mode de diffusion de leurs chansons et sur la constitution des chansonniers. Cependant, elles ont toujours paru donner des poèmes eux-mêmes une lecture appauvrie et réductrice. Y chercher une idée de la poésie, voire une herméneutique de la poésie des troubadours, paraît non seulement une gageure, mais même une provocation, celle que constituerait la réhabilitation d'une critique biographique, puisque ces textes réduisent systématiquement les chansons aux circonstances supposées de leur composition. Et pourtant, vidas et razos ont su, à leur façon, mettre en évidence des traits essentiels de la poésie des troubadours. Tout l'effort du cours a été pour le montrer.

Avant de se lancer dans cette démonstration, on a rappelé les données fondamentales de l'histoire littéraire et de la philologie touchant la constitution des chansonniers occitans. Pour que les chansons des troubadours soient parvenues jusqu'à nous, il faut bien qu'elles aient été écrites. Quel qu'ait été leur mode primitif de diffusion - tantôt orale, par la voix de jongleurs ou des poètes euxmêmes, tantôt écrite sur de petits rouleaux de parchemin —, elles ont été rassemblées à une date relativement tardive, à la fin du XIII ${ }^{\mathrm{e}}$ (cinq manuscrits) et au 
$\mathrm{XIV}^{\mathrm{e}}$ siècle (une quinzaine de manuscrits), c'est-à-dire au moment où l'art des troubadours s'épuisait, dans des manuscrits anthologiques, généralement soignés ou luxueux, les chansonniers. C'est dans ces manuscrits, ou du moins dans un bon nombre d'entre eux, que figurent les vidas, qui relatent la vie des troubadours, et les razos, qui présentent et commentent certains de leurs poèmes. Elles sont plus de deux cents et concernent une centaine de troubadours. Certaines se réduisent à une biographie de deux ou trois lignes. D'autres s'étendent sur des pages et forment comme un petit roman du poète.

Comment se sont-elles retrouvées dans ces manuscrits? Qui les a composées et pour quel usage? Il semble bien qu'il s'agisse au départ d'une sorte de boniment, d'explication préalable, de prélude divertissant en guise de captatio benevolentiae, que le troubadour ou le jongleur débitait avant d'interpréter une chanson. Ainsi, la vida de Guilhem de la Tor, qui, dit-elle, devint fou à la mort de son amie, ne voulait pas croire qu'elle fût morte et cherchait à la ressusciter, nous apprend que «lorsqu'il voulait dire ses chansons, il consacrait au commentaire un discours plus long que la chanson même ». D'où leur ton volontiers détaché, humoristique, moqueur. Mais les vidas et les razos rassemblées dans les chansonniers ont probablement été écrites en série, peut-être par un nombre d'auteurs restreint, comme Uc de Saint-Circ, troubadour originaire du Quercy, mais vivant en Italie, qui signe la vida de Bernard de Ventadour et les razos de Savaric de Mauléon, ou Miquel de la Tor, auteur de la vida de Peire Cardenal, qui se dit «écrivain » et dont on a pu tenter de reconstituer le «livre». Parfois l'auteur se met en scène sans se nommer: celui de la vida de Cadenet certifie qu'il a eu une connaissance directe des faits et gestes du troubadour.

Parfois, vidas et razos sont regroupées dans une section particulière du recueil, au début du manuscrit, comme dans $\mathrm{R}$, à la fin, comme dans $\mathrm{E}$, ou au milieu, comme dans P. Mais la disposition la plus caractéristique, que l'on a pu désigner comme «la forme chansonnier» (Maria Luisa Meneghetti), est celle que l'on trouve dans des manuscrits copiés en Vénétie. Les chansons de chaque troubadour, écrites à l'encre noire, sont précédées de sa vida, écrite à l'encre rouge, et, pour certaines, de leur razo, également écrite en rouge. L'alternance des textes narratifs en prose et des poèmes est ainsi matériellement signalée. Comme l'a souligné Fabio Zinelli, cette disposition est certainement celle en vue de laquelle les vidas ont été composées, puisqu'elles se terminent habituellement par des formules comme «Et il fit les chansons que vous allez entendre et qui sont écrites ci-dessous » (Bernard de Ventadour), « Et ici sont écrites un grand nombre de ses chansons »(Guiraut de Borneil et al.), «Et des chansons de lui sont ici écrites », «Et il composa les chansons qui sont transcrites ici » (Élias de Barjols), «Il composa maintes bonnes chansons, dont quelques-unes sont écrites ici, comme vous entendrez » (Raimon Jordan). Au contraire, s'agissant des razos, la formule habituelle «Et il fit à ce sujet la chanson (le sirventès) qui dit... » n'implique pas que la chanson soit intégralement citée à la suite. De fait, certaines des razos les plus développées se trouvent dans des chansonniers qui n'adoptent 
pas la «disposition vénète », comme $\mathrm{R}$ ou $\mathrm{P}$, ce dernier, cependant, citant souvent à la suite de la razo une strophe entière, et non pas le seul premier vers.

On s'est posé la question de savoir si la disposition qui consiste à faire alterner vidas et razos en prose avec les chansons permet de considérer les chansonniers comme des prosimètres. Cette qualification paraît inappropriée, car le chansonnier n'est pas en lui-même une œuvre littéraire cohérente, mais une collection de poèmes divers et indépendants. L'introduction de textes en prose répond à une intention purement didactique, visant à donner aux poèmes qui se succèdent dans le manuscrit un éclairage biographique. À ce compte, on peut qualifier de prosimètre n'importe quelle anthologie poétique dotée d'une introduction et de notes.

Nous définissons le prosimètre comme une composition où alternent passages en prose et passages en vers - le modèle du genre, aux yeux du Moyen Âge, étant la Consolation de Philosophie de Boèce. Il est vrai que ce sens se répand, puis s'impose, mais seulement à partir du XIII ${ }^{e}$ siècle. Chez les théoriciens antérieurs, comme l'a montré Anne-Marie Turcan-Verkerk, le prosimètre se définit différemment. Pour Hugues de Bologne, le prosimetrum ne fait pas alterner les passages en prose et les passages en vers : il mêle le vers à la prose. D'autre part, certains auteurs placent dans une même catégorie la prose et le vers rythmique, par opposition au mètre, ce qui n'a, au fond, rien de surprenant et ce que confirme la liturgie, qui appelle «prose » un poème rythmique. On observe que dans le domaine vernaculaire, qui ignore la poésie métrique et ne connaît que la poésie rythmique, seule existe la première catégorie, celle de la prose et du vers rythmique, associés en latin. Écrire un ouvrage associant prose et vers rythmiques, c'est, du point de vue du latin, écrire un ouvrage (relativement) homogène. Cette proposition est, bien entendu, excessive, ne serait-ce que parce que tout système tend vers une opposition binaire : l'un des termes de l'opposition disparu, celle-ci tend à se reformer par clivage du terme restant. La disparition en roman de la poésie métrique a eu pour conséquence d'opposer définitivement la prose à la poésie rythmique. C'est en effet ce qui s'est passé, mais accompagné d'autres phénomènes qui compliquent et brouillent les choses. Avant le développement tardif de la prose, on constate bien la relative unité d'une langue littéraire rythmée selon diverses modalités, ces modalités se définissant en fonction de la prosodie (types de vers et de strophes), mais plus fondamentalement en fonction de la musique ou de la musicalité de l'interprétation (chant, cantillation, lecture rythmée). Ce sont ces catégories de «musicalité » qui définissent les inévitables systèmes d'opposition.

Pour en revenir aux chansonniers occitans, on constate que dans la «forme chansonnier » vénéto-sicilienne, disposition première et naturelle, comme l'impliquent les formulations mêmes des vidas qui supposent leur enchaînement avec les poèmes, le contraste entre la prose et les chansons est visuellement marqué par l'alternance des encres rouge et noire. Il ne l'est guère autrement. L'usage général des manuscrits médiévaux, on le sait, est d'aller à la ligne à la fin de chaque vers dans tous les cas, qu'il s'agisse de laisses épiques, de couplets d'octosyllabes, de dits divers et variés etc., sauf pour la poésie lyrique, dont les 
vers sont copiés en continu. Peut-être cette exception a-t-elle à voir avec la notation musicale, puisque au Moyen Âge comme aujourd'hui, le texte chanté est copié en continu sous la portée (ou, avant le $\mathrm{XI}^{\mathrm{e}}$ siècle, sous la ligne de neumes). Mais les chansonniers vénètes ne donnent pas la mélodie. Sans elle, la disposition des chansons est purement et simplement celle de la prose. Le passage d'une strophe à l'autre est seul marqué par un alinéa et par une initiale colorée. Certes, les vers sont séparés par un point, mais des points scandent aussi la prose des vidas et des razos - cette prose particulière que sa simplicité et son uniformité rendent rythmée et chantante. Ces chansonniers sont donc des prosimètres au sens habituel du terme. Ils le sont matériellement, mais aussi par le sens et les effets que produit l'enchaînement des vidas et des poèmes, un sens et des effets nouveaux, qu'on ne trouverait ni dans la vida ou la razo seule ni dans la chanson seule, mais qui naissent de l'association et de la confrontation de l'une et de l'autre. Mais ils sont aussi des prosimètres au sens d'Hugues de Bologne, puisque, en même temps qu'ils la soulignent, ils réduisent jusqu'à un certain point l'opposition du vers et de la prose. Vidas et razos ne sont pas des poèmes. Mais, loin d'être en perpétuel décalage, voire en perpétuel contresens au regard des poèmes des troubadours, elles les prolongent et en approfondissent les résonances.

Leur intérêt vient moins de la petite partie de vérité qu'elles contiennent touchant la biographie des poètes que de la grande part d'imagination qui les a engendrées. Cette imagination, nourrie par les poèmes qu'elles prétendent expliquer, est comme la transposition sur le mode narratif d'une appréhension poétique du monde, comme si elle parcourait en sens inverse le chemin de l'imagination poétique. Prendre conscience de ce cheminement, c'est écarter la tentation de traiter vidas et razos avec condescendance, comme on l'a presque toujours fait.

On les a traitées avec condescendance pour plusieurs raisons. D'abord, parce qu'on les a considérées comme des documents, qu'on y a donc cherché des renseignements historiques fiables et qu'on s'est irrité que ceux qu'elles donnaient ne le fussent point. On leur a donc reproché leur démarche même : inventer leur matière en brodant sur un mot ou un détail empruntés au poème. On leur a aussi reproché leur style monotone et convenu, sans en goûter le charme surprenant, ou plus probablement sans s'avouer qu'on le goûtait. Le style et le mode de la narration sont, de fait, si codés que vidas et razos sont extrêmement faciles à pasticher, comme l'avait observé Jean Boutière, leur éditeur. Réduite à sa plus simple expression, une vida peut se présenter ainsi :

Pierre Brémon le Tort était un pauvre chevalier du Viennois. Il était bon troubadour et honoré par toute la bonne société.

Ou encore, avec déjà le germe d'un récit :

Guilhem de Montaignagol était un chevalier de Provence; il était bon troubadour et grand amoureux. Il courtisait Madame Jausserande, du château de Lunel; et il fit pour elle maintes bonnes chansons. 
Si la vida brodait sur le thème des amours de Guilhem et de Jausserande, si une razo rattachait l'une de ses chansons à une péripétie de cet amour - brouille, infidélité, malveillance des envieux, vengeance d'un mari jaloux — on se trouverait dans le cas des vidas et des razos amplifiées et développées. Mais, si longues et si riches en péripéties qu'elles puissent être, vidas et razos sont toutes fidèles à cette narration grêle, où des phrases brèves, presque dépourvues de subordination, s'enchaînent, uniformément liées les unes aux autres par la conjonction «et ». Pourquoi ces récits, en apparence sans art, exercent-ils un tel charme ? Parce que leur ton uni, égal, ennemi de toute démonstration et de tout effet appuyé, fait ressortir par contraste, selon les cas, le piquant, l'humour, le tragique ou la complexité psychologique de ce qu'ils racontent, de la même façon qu'une histoire amusante gagne à être contée sur un ton pince-sans-rire, une histoire poignante sur un ton détaché.

Mais n'est-ce pas leur faire trop de crédit que de leur prêter une telle richesse ? Complexité psychologique, n'est-ce pas beaucoup dire ? Car la raison la plus sérieuse de mal juger les razos est qu'elles paraissent faire une lecture pauvre et une pauvre lecture de poèmes très riches. Ces poèmes difficiles et tendus, elliptiques et allusifs, qui évitent si soigneusement de tomber dans l'anecdote, qui aspirent si ardemment à la généralité (généralité au demeurant nécessaire à une chanson, que chacun doit pouvoir s'approprier), elles en rendent compte en les réduisant à des circonstances biographiques et de ce seul fait paraissent commettre un contresens sur la poétique qui les fonde.

Ce trait paraît tenir pour une part au décalage chronologique et à l'évolution de la poésie. Vidas et razos, qui sont pour l'essentiel des textes du XIII' siècle, portent sur des poètes dont les plus importants ont vécu au XII ${ }^{\mathrm{e}}$ siècle et qui tous, même les plus tardifs, s'inscrivent dans la continuité d'un système poétique élaboré à cette époque. Or, au XIII ${ }^{\mathrm{e}}$ siècle, la poésie, connaît une mutation que j'ai souvent décrite. À côté du lyrisme chanté, essentiellement amoureux, se développe une poésie récitée, à l'origine édifiante, morale ou satirique. Ses auteurs éprouvent généralement le besoin de se mettre en scène ou d'intervenir en leur nom propre pour témoigner de la vérité de leur propos. Cette tendance transforme rapidement cette poésie en une poésie du moi. Les poètes ne visent plus une idéalisation du moi, répondant à un idéal de l'amour, comme les troubadours et leurs émules. Ils se livrent au contraire à une sorte de théatralisation anecdotique du moi, offrant d'eux-mêmes un portrait souvent dépréciatif, qui tourne volontiers à la charge caricaturale. C'est le mouvement qui, amorcé en France à la fin du XII ${ }^{e}$ siècle, trouve à la fin du XIII ${ }^{e}$ siècle son expression la plus caractéristique avec Rutebeuf, mais dont il existe l'équivalent dans l'Europe entière. C'est à ce moment et dans ce contexte que vidas et razos sont composées et recueillies. Elles ont donc pu subir l'influence de cette nouvelle conception poétique, largement fondée sur l'anecdote et sur la confidence, ou sur la fausse confidence, biographique, et l'appliquer de façon inappropriée à des poèmes relevant d'une conception toute différente. 
Mais l'ont-elles vraiment fait de façon inappropriée? Si on les lit avec une attention bienveillante, on constate qu'elles perçoivent souvent avec acuité le point essentiel des poèmes qu'elles commentent, mais qu'elles en rendent compte par l'extrapolation narrative, et non par l'analyse critique qui nous est naturelle. Elles concentrent leur exégèse sur quelques mots, et leur exégèse est un récit. Quoi d'étonnant? L'exégèse théologique de l'époque, sous sa forme didactique, ne consiste-t-elle pas à isoler quelques mots de l'Écriture et à en épuiser le sens ? Et que l'exégèse prenne la forme d'un récit et non celle d'un exposé abstrait, n'est-ce pas là le principe même de l'enseignement du Christ par paraboles?

Restait à montrer que les razos sont capables de mettre le doigt sur le point crucial (généralement le point douloureux) et sur l'effet majeur des chansons. C'est ce qu'on a fait à travers une série d'exemples, dont le premier était le plus banal mais aussi celui qui, grâce à la brièveté des textes en prose, peut le plus aisément figurer dans un résumé. Il est fourni par la brève razo de la plus célèbre chanson du plus illustre des troubadours, la chanson de l'alouette de Bernard de Ventadour. Cette razo se fonde sur la fiction propagée par la vida composée par Uc de Saint-Circ, que le troubadour était épris d'Aliénor d'Aquitaine, désignée comme la duchesse de Normandie :

Et Bernart l'appelait « Alouette », à cause d'un chevalier qui l'aimait et qu'elle appelait

«Rayon ». Et, un jour, le chevalier vint auprès de la duchesse et entra dans la chambre.

La dame, qui le vit, leva alors le pan de son manteau et le lui mit sur le cou; et elle se laissa choir sur le lit. Et Bernart vit tout, car une suivante de la dame le lui montra en cachette; et sur ce sujet, il fit alors la chanson qui dit:

Quand je vois l'alouette agiter...

Cette razo est une simple extrapolation à partir des trois premiers vers de la chanson:

Can vei la lauzeta mover

De joi sas alas contra'1 rai,

Que s'oblid'e s laissa chazer...

Quand je vois l'alouette agiter

de joie ses ailes face au rayon (de soleil), puis s'oublier elle-même et se laisser choir...

Elle est unanimement jugée pitoyable, et cela pour deux raisons. La première est que, non contente de tirer, selon l'usage, sa matière de la chanson qu'elle commente, elle le laisse voir maladroitement : «Il l'appelait Alouette à cause d'un chevalier qui l'aimait et qu'elle appelait Rayon ». En voilà, une raison! En quoi le fait qu'Aliénor appelle le chevalier Rayon explique-t-il que Bernard l'appelle Alouette ? Pourquoi l'un des deux mots appelle-t-il l'autre? Quel rapport y a-t-il entre un rayon et une alouette? Aucun, sinon que les deux mots sont associés au début de la chanson.

La seconde raison qui déconsidère cette razo est plus grave. La gloire de la chanson de l'alouette n'est pas usurpée. Sa première strophe est admirable. L'accumulation des éléments habituels de l'incipit printanier y est remplacée par l'image épurée, unique, de l'alouette à contre-jour, dans un rayon de soleil. À 
peine une image (on voit mal à contre-jour, et l'alouette est si petite - un point face au soleil), mais plutôt une sorte de représentation presque abstraite du mouvement. Et quel mouvement! Ce geste suicidaire - se laisser tomber par « oubli de soi-même» - qui évoque immédiatement pour le poète la petite mort de la jouissance amoureuse, où la raison s'abolit, et fait naître en lui «l'envie de tous ceux qu'il voit jouissant». Ce mouvement et cette chute, qui n'est pas une chute, mais une exaltation, rendus par les enjambements mover / de joi ou ai! tan grans enveya m'en ve / de cui qu'eu veya jauzion (on croit que le poème envie l'alouette, mais le vers suivant révèle qu'il envie «quiconque il voit jouissant », et c'est la même chose); rendus aussi, dans la mélodie, par la chute de la voix, en un ambitus inhabituel, sur le ve de ai! tan grans enveia m'en ve.

Là-dessus, la razo invente une histoire grotesque de petit jeu érotique entre Aliénor d'Aquitaine et son amant, tandis que le poète les épie par le trou de la serrure avec la complicité de la bonne. C'est pitoyable. Soit. Mais la razo n'en a pas moins saisi l'essentiel, et elle sait dire l'essentiel. L'essentiel, c'est la jalousie sexuelle et le voyeurisme: «quelle envie me vient de quiconque je vois jouissant». La razo ne dit pas autre chose. On dira qu'elle ne commente pas l'imbrication des images, des mouvements affectifs et des associations mentales, les jeux de la forme métrique, les effets de la mélodie. C'est un récit, ce n'est pas une explication de texte. Est-ce à dire que ce récit est insensible à la poésie ? Non, puisqu'il en ressent exactement et violemment l'effet et le sens. Non encore, puisqu'il ne se dispense pas de citer le poème. L'ensemble des razos et des poèmes constitue bien un prosimètre en ce que tous les éléments sont nécessaires à l'ensemble. Le récit en prose n'a nullement la prétention de se substituer au poème. Il ne cherche qu'à le mettre en valeur, sensible qu'il est à ce qu'il a d'irremplaçable.

Or, cette jalousie, cette frustration, cette irritation de la sexualité par le voyeurisme et par la comparaison avec le sort d'un amant plus fortuné, voilà ce que les récits des razos développent avec prédilection, et à juste titre, car ce n'est pas seulement le thème privilégié des troubadours, c'est la racine même de leur perception poétique du monde et de la présence du monde dans leur poésie. C'est la racine de leur expérience de l'amour et de leur représentation de l'amour comme expérience poétique.

Un commentaire du mot «envie» dans les vers de la chanson de l'alouette cité plus haut, en relation avec le désir d'une part, la jalousie de l'autre, a conduit à analyser une chanson de Raimbaut de Vaqueiras (Ja non cuidei vezer) où l'on trouve un emploi particulier, à la fois énigmatique et éclairant, de l'adjectif « envieux»: lorsqu'un cœur sauvage succombe à l'amour, il sait mieux aimer sa dame c'umilz trop amoros / de totas envejos, qu'un humble trop amoureux, envieux de toutes — c'est-à-dire « qui les désire toutes », mais « envieux » ajoute des ambiguïtés de toute sorte. Celui qui est «trop amoureux » les désire toutes, mais il est aussi envieux de toutes. Il voudrait jouir de toutes parce qu'il est 
envieux de la jouissance de toutes. C'est pourquoi il est «humble»: il éprouve violemment cette infériorité de ne pas jouir avec toutes, alors que toutes ont la capacité de jouir (ce qui est peut-être la définition même du donjuanisme). De même, dans la chanson de l'alouette, on peut considérer que le poète est envieux de la jouissance éprouvée par celle qu'il aime autant que de la jouissance qu'elle donne à un autre.

Or, il se trouve que cette chanson de Raimbaut de Vaqueiras a donné lieu à une curieuse razo qui n'est pas sans analogie avec celle de la chanson de l'alouette, au moins par le rôle qu'y joue le voyeurisme. Raimbaut, pauvre chevalier protégé par le marquis Boniface de Monferrat, est amoureux de la sœur du marquis, Béatrice. Il l'épie continuellement par un soupirail de sa chambre. Un jour il voit le marquis, au retour de la chasse, entrer dans la chambre et poser une épée contre le lit. Restée seule, Béatrice ôte son surcot, reste en robe de dessous, tire l'épée du fourreau et s'escrime avec elle. Puis elle la remet dans son fourreau. Raimbaut lui donne alors le senhal (surnom) de Beau Chevalier, comme dans la chanson Ja non cuidei vezer...

On a conclu un long commentaire de cette razo en observant que, dans la scène qu'elle décrit, la dame prend des attitudes masculines, manie un objet essentiellement masculin, et reçoit pour cela un senhal masculin. L'érotisation métaphorique de la scène va de pair avec la masculinisation de la dame. Celleci se rend désirable pour l'homme en se faisant semblable à lui. N'est-ce pas une interprétation pénétrante des mots «envier» et «envieux» tels qu'ils sont employés dans les poèmes et de l'infériorité qui frappe «l'humble trop amoureux envieux de toutes »? L'attitude de ce Don Juan comme l'hésitation du poète dans la chanson de l'alouette supposent une identification des deux jouissances, et donc une ressemblance entre l'homme et la femme qu'il désire. S'il veut aimer, l'homme doit se féminiser : Hercule file aux pieds d'Omphale et le troubadour se fait humble, doux, obéissant (qualités féminines), tandis que sa dame est désignée au masculin comme mi dons (meus dominus, mon seigneur), reçoit un senhal masculin, le «domine», exerce sur lui le pouvoir de l'homme. En ce sens, la razo, avec ce récit d'une femme qui joue à l'homme sous le regard d'un homme, éclaire et prolonge les vers de Raimbaut. Certes, le troubadour proteste dans son poème qu'il n'est pas comme les autres, «envieux de toutes ». Mais c'est seulement pour dire qu'il est envieux d'une seule et qu'il « revient de loin ». Pourtant, il est bel et bien revenu : d'estraing, dit la chanson, il est devenu privat. Dans l'ombre, humblement collé à son soupirail, il regarde la femme qu'il aime manier l'épée.

Cette image, c'est la razo qui nous l'offre. Elle est étrangère au poème. L'ambiguïté d'enveya et d'envejos, ambiguïté certes présente dans les deux chansons, mais comme une simple suggestion, ce sont les razos qui la mettent en évidence et qui en exploitent les résonances. C'est dire qu'elles donnent à voir l'essentiel. Car toute la poésie des troubadours se fonde sur cette ambiguïté ou sur cette hésitation touchant l'objet de l'envie : «avoir envie de » ou « être envieux de »; 
avoir envie de la personne aimée, c'est-à-dire la désirer, ou être envieux d'elle, ou encore être envieux de celui qui la possède (dont l'envie d'elle est satisfaite). Ce sont trois envies différentes, et c'est la même envie. Tout le noud de la jalousie et de l'irritation sexuelle est là.

De fait, comme on l'a dit, beaucoup d'autres razos rapportent explicitement les poèmes qu'elles commentent à des situations de frustration, de jalousie, d'excitation sexuelle liée à la jouissance de l'autre et, comme si les auteurs des razos avaient lu René Girard, de désir mimétique et de rivalité mimétique. Elles ne se lassent pas de mettre en scène L'éternel mari, qui ne peut désirer sa femme que s'il la voit désirée par d'autres et si le désir des autres justifie le sien à ses propres yeux, ou sa variante, celle de l'homme qui tombe toujours amoureux de femmes qui ne sont pas libres parce qu'il a lui aussi besoin que le choix d'autrui le conforte dans le sien. Elles imaginent d'autant plus volontiers de telles situations que celles-ci caractérisent souvent les amours adolescentes, extrêmes, peu sûres d'elles-mêmes, cherchant parfois l'assurance qui leur manque dans la femme qui en est l'objet, en la choisissant plus âgée et plus expérimentée, et que l'amour célébré par les chansons des troubadours a souvent les traits d'un amour adolescent : à la fois audacieux et timide, brûlant et réservé, craignant sans cesse de déplaire à celle qui en est l'objet, porté à une sorte de voyeurisme respectueux, débordant d'une imagination torride et paraissant toujours s'arrêter au bord de l'acte sexuel lui-même, ou du moins le couvrir d'une gaze.

On a d'abord illustré ce trait avec la vida et les razos de Raimon de Miraval. La pauvreté de ce chevalier de la région de Carcassonne le contraint, pour se faire une place dans la bonne société, à faire usage de ses talents et de son charme auprès de ses protecteurs comme auprès des femmes. Sa vida, conservée dans dix manuscrits, le présente comme un arbitre des élégances et de la vie courtoise, recherché à ce titre par les dames, qui veulent toutes être courtisées et célébrées par lui, sans qu'aucune lui accorde jamais rien. Il n'a donc luimême de valeur que comme objet du désir mimétique : ce qui est désirable en lui, ce n'est pas lui, mais le fait que les autres le désirent. Sous une apparence flatteuse, sa vida trace de lui un portrait au fond désobligeant : celui d'un personnage qui fait l'officieux, se rend indispensable et fait sa fortune, certes par ses qualités de poète, mais aussi par son talent à colporter les commérages sur les amours des uns et des autres, peut-être même par ses talents d'entremetteur (c'est ce que telle de ses chansons peut suggérer, même si la razo qui lui est attachée lui donne un sens différent), certainement par sa capacité à faire le joli cœur auprès des dames, à s'en éprendre ou à s'en dire épris, et à les flatter.

Parmi toutes les razos que l'on a commentées, l'une, conservée dans les chansonniers E, P et R, a particulièrement retenu notre attention. Elle montre Raimon de Miraval épris d'Azalaiis de Boissezon, qui, comme les autres, l'accueille fort bien, «jugeant qu'il pouvait lui donner plus de prix et d'honneur que nul homme au monde ». Elle a bien jugé. Il la célèbre tant dans ses poèmes que tous les plus grands seigneurs s'éprennent d'elle, et surtout le roi Pierre II 
d'Aragon, que Raimon invite, dans la strophe autour de laquelle la razo est élaborée, à venir faire sa cour auprès d'Azalaïs en lui promettant une grande joie. Le roi, séducteur notoire, se rend à cette invitation et la dame, dès le premier soir, lui accorde ses faveurs. Et Raimon de Miraval, qui attendait du roi qu'il intercédât pour lui auprès d'Azalaïs, en est très triste.

Il y a, dans ce récit, une contradiction criante entre l'intention avouée du poète (ménager une entrevue entre le roi d'Aragon et Azalaïs pour que le roi puisse plaider auprès d'elle sa cause à lui, Miraval) et le résultat prévisible de la démarche. Une contradiction qui n'est pas seulement entre la razo et la strophe qu'elle cite, mais entre cette strophe, qu'on peut rapprocher d'un sirventès du même poète, et le reste de la chanson, comme l'auteur de la razo l'a, semble$\mathrm{t}$-il, perçu. Une contradiction d'autant plus grande que Miraval s'efforce par ses chansons d'éveiller l'amour du roi et que la strophe qui fonde la razo en est un exemple. La contradiction est telle qu'on soupçonne que le poète, dans sa chanson, joue effectivement et délibérément le rôle d'entremetteur et que la razo enrichit le poème d'une possibilité de souffrance qui n'y est pas, mais qui est la vérité cachée de ce genre de situation. Le poème n'a d'intérêt, de profondeur, de résonances, que s'il dissimule la situation de «l'éternel mari ». Une fois de plus, la razo met le doigt sur l'essentiel : son récit livre la vérité poétique de la chanson. Chacun ne peut jouir qu'en imaginant ce que serait la jouissance d'un autre à sa place, et cette imagination est une occasion de souffrance. Vidas et razos ne se lassent pas de méditer cette leçon et d'exploiter les possibilités infinies de souffrance et d'humiliation qu'elle ouvre.

D'autres récits consacrés à Raimon de Miraval, mais surtout la vida et les razos de Gaucelm Faidit en ont fourni l'illustration : celle dans laquelle on voit Audiarde de Malemort inventer un stratagème qui permet à Marie de Ventadour de se débarrasser de ce soupirant encombrant, celle qui montre le malheureux Gaucelm contraint, sans avoir rien fait de mal, d'implorer le pardon de Jordane d'Embrun. Mais surtout celle de la chanson Si anc nuls hom per aver fin coratge : Gaucelm aime la vicomtesse Marguerite d'Aubusson et s'en croit aimé, bien qu'elle ne lui ait jamais rien accordé, sinon d'avoir souffert un jour qu'il lui baisât le cou. En réalité, elle aime Hugues de Lusignan, qui est un grand ami de Gaucelm Faidit. Elle feint d'être malade et déclare qu'elle veut faire le pèlerinage de Rocamadour. Elle donne un rendez-vous secret à Hugues de Lusignan à Uzerche, qui est sur le chemin, dans la maison même de Gaucelm Faidit. La femme de celui-ci les y reçoit très bien et les amants y passent deux nuits délicieuses à l'aller et au retour du pèlerinage. Quand Gaucelm revient chez lui, il apprend tout, en est si triste qu'il veut mourir et compose cette chanson, «la dernière qu'il fit ». Il n'y aurait guère là qu'un sujet de fabliau sans cet extraordinaire raffinement dans le sadisme. Sadisme des amants, mais aussi sadisme - excusable — de la femme de Gaucelm, que la razo a l'habileté de ne pas commenter. Les historiens de la littérature ont reproché à la razo d'affirmer que cette chanson est la dernière composée par Gaucelm Faidit, ce 
qui n'est certainement pas le cas. Peut-être a-t-elle eu le sentiment que Gaucelm, qui a connu tant de péripéties et de déconvenues amoureuses, ne pouvait survivre poétiquement (et à peine survivre tout court) à cet excès du poison de l'humiliation sexuelle, qui, à dose raisonnable, est l'aliment de la poésie.

On a enfin approfondi la lecture faite par les razos des chansons des troubadours dans la perspective du désir et de la rivalité mimétiques par l'analyse des deux razos les plus significatives à cet égard, celle de Pons de Chapteuil et surtout celle de Guilhem de Balaun.

Beaucoup de ces récits peuvent être lus dans une perspective sociologique, celle des travaux de Erich Köhler, inspirés par Marc Bloch, celle de Maria Luisa Meneghetti, lisant vidas et razos à la lumière de Köhler : le troubadour de rang inférieur, Gaucelm Faidit ou Raymond de Miraval, est trompé et sexuellement humilié par le grand seigneur avec lequel il est en rivalité. Une telle lecture s'impose, bien sûr. Mais les razos elles-mêmes évitent cette conclusion, que leur récit rend pourtant pratiquement explicite. Ce qu'elles disent est d'un autre ordre, et peut-être plus profond. L'interprétation sociologique à la Köhler n'est pas le fin mot d'une lecture des razos, mais son point de départ. Décrire une situation où le troubadour est trompé et vaincu par le grand seigneur, ce n'est pas, de la part du critique, se livrer à un décryptage sociologique, c'est, de la part de la razo, utiliser une situation qui va de soi (bien sûr que le grand seigneur gagne toujours !) pour faire sentir l'exacerbation d'une sexualité frustrée, humiliée, jalouse, taraudée par le voyeurisme. La vérité ultime de la poésie du désir qu'est celle des troubadours - poésie du désir sous le regard de l'autre et poésie du désir de la jouissance de l'autre, ce ne sont pas les conditions sociales dans lesquelles elle s'inscrit. C'est le contraire : la vérité que l'on fait jaillir en s'aidant de rapports sociaux qui parlent d'eux-mêmes et n'ont pas besoin de commentaire, c'est celle du désir.

Tout cela, les razos le disent. Elles mettent les points sur les i, là où les poèmes sont évanescents et vagues. Et ainsi, elles jouent réellement leur rôle de commentaires critiques. En injectant l'anecdote dans la chanson, elles font apparaître que la chanson en elle-même est dénuée d'anecdote, qu'elle est lisse, ou plutôt que ses seules aspérités sont celles du langage, qu'elle paraît s'inscrire perpétuellement dans le topos de la plainte amoureuse et qu'elle ne s'en évade ou n'y met du ragoût que subrepticement, sournoisement, mais d'une façon qui n'échappe pas à la razo, heureuse alors d'en rajouter. En racontant, elles font ainsi ressortir par contraste ce qu'est une poétique qui exclut le récit. En s'opposant aux chansons, en se posant en complément nécessaire aux chansons, elles développent sous forme narrative les implications d'une poétique, mais elles font ressortir aussi, a contrario, ce qu'est une poétique d'où le récit est absent, puisqu'elles sont au service des chansons.

Ces remarques ont permis d'aborder pour finir la question de l'attention portée par les razos au langage et à la création poétique en eux-mêmes. Sont-elles 
capables d'autre chose que d'expliciter sous la forme de l'exemplum un contenu affectif ou psychologique latent, que le poème désigne par fulgurance dans le raccourci de l'allusion ou de l'ellipse ? N'y a-t-il pas des razos de l'acte poétique lui-même ? Il y en a une, mais une seule, extrêmement célèbre et fort étrange : non une genèse, mais une anti-genèse, un récit de la non création poétique par laquelle la création poétique advient pourtant. C'est la razo de la chanson d'Arnaut Daniel, Anc ieu non l'aic, mas elha m'a / Tos temps en son poder Amors. Au terme d'une analyse trop complexe pour être aisément résumée, on a montré comment l'anecdote amusante de la razo s'invente à la faveur d'un détournement de la chanson au profit d'une réflexion sur la création poétique. L'apparente injustice de Richard Cœur de Lion qui «donne » à Arnaut la chanson composée par le jongleur se fonde sur le paradoxe implicite selon lequel Arnaut Daniel ne peut pas être l'auteur de la chanson parce qu'il est le seul à pouvoir en être l'auteur - et de fait, il l'est.

Après avoir fait valoir ce que la nouvelle à l'italienne, ancêtre du roman moderne, doit aux razos, la conclusion a insisté sur ce qu'elles ont à nous apprendre touchant les chansons des troubadours, mais aussi la poésie en général. D'une part, elles montrent en racontant que le poème ne raconte pas. En explicitant sous la forme du récit les obsessions du poème, elles attirent l'attention sur le fait que le poème ne les communique pas sous la forme du récit. Mais d'autre part, elles montrent aussi qu'il y a dans le poème quelque chose qui appelle le récit. Dire que les chansons des troubadours refusent l'anecdotique est inexact. Elles sont remplies de noms propres et de noms de lieux, d'allusions à des personnages et à des circonstances. Leur tornada les insère presque toujours dans le champ des échanges sociaux. Ces référents multiples ne s'insèrent pas dans un discours cohérent qui justifierait et expliciterait leur présence. Ils émergent de façon visible et décalée, charriés par le courant du poème, animé qu'il est par un effort et une urgence qui n'ont rien à voir avec eux : ceux de faire coïncider les tensions du langage et celles de l'amour. Et pourtant ils sont là. Les razos s'accrochent à eux, montrent à partir d'eux que ces tensions de l'amour peuvent se dire autrement, par le récit de l'amour, mais du coup attirent aussi l'attention sur le fait que le poème les dit à sa manière, sans le détour du récit, mais en insistant, par le recours aux référents, sur le fait que le récit pourrait exister. En ce sens, les razos obéissent aux injonctions du poème en se séparant de lui. Elles le mettent en valeur par contraste. Enfin, on a souligné que les vidas et les razos ne constituent pas un phénomène unique et que la littérature universelle, à commencer par les romans «médiévaux» japonais, fournit de nombreux exemples de récits qui donnent la clé des poèmes qu'ils enchâssent.

Le séminaire, sur le thème «Transpositions, circulations et métamorphoses du texte médiéval », a accueilli, outre la séance d'ouverture du professeur et son exposé sur les implications poétiques du manuscrit Chantilly, Condé 388, six invités. Le 20 février 2007, David Hult, professeur à l'université de Californie à Berkeley : «Poésie et traduction du savoir chez Jean de Meun ». Le 27 février, 
Pilar Lorenzo Gradín, professeur associé à l'université de Saint-Jacques de Compostelle : «Le deuxième texte dans les chansonniers galégo-portugais ». Le 6 mars, Richard Trachsler, maître de conférences à l'université de ParisSorbonne, membre de l'I.U.F. : « Histoires d'une vie. Regards sur les manuscrits de divination médiévaux ». Le 13 mars, Jeffrey Rider, professeur à l'université Wesleyan : «Le poème comme récit familial. La mère de Perceval ». Le 20 mars, Doris Ruhe, professeur à l'université de Greifswald : «Stratégies de l'intime. La lettre et le secret dans la littérature narrative du Moyen Âge français ». Le 5 avril, Agathe Sultan, maître de conférences à l'université de Bordeaux III : "Métamorphoses de la double ballade à la fin du Moyen Âge ».

Six heures de cours ont été délocalisées à l'université Harvard, à l'université de Sienne et à l'université de Poitiers.

Une séance de séminaire, qui devait être délocalisée à l'université d'Oxford au mois de mai, a dû être repoussée à l'automne.

Toutes les heures de cours données à Paris ont été diffusées par France-Culture dans le cadre de l'émission «Éloge du savoir».

\section{ACTIVITÉS DE LA CHAIRE}

\section{Activités des collaborateurs}

Odile Bombarde, maître de conférences

«La pensée du rêve", communication au colloque "Yves Bonnefoy, poésie et savoirs » du Centre culturel international de Cerisy-la-Salle (29 août 2006).

Communications au séminaire «La qualification du transfert » de l'Association psychanalytique de France, Paris (17 octobre, 21 novembre et 19 décembre 2006).

Catherine Fabre, maître de conférences, Pierre Leroy, maitre de conférences honoraire.

Publication :

Édition annotée de Charles-Louis-Marie de Coskaer, comte de La Vieuville. Mémoires et ouvres diverses, préface de Marc Fumaroli, Paris, Champion, 2006, 352 pages.

\section{Andrea Valentini, ATER}

Publications :

«Le remaniement de Gui de Mori et sa tradition manuscrite», dans De la Rose. Texte, image, fortune, éd. Catherine BeL et Herman BraEt, Louvain, Peeters, 2006 (Synthema, 3), pp. 299-320.

«Le rossignol et la grenouille», dans Studi di Filologia romanza offerti a Valeria Bertolucci Pizzorusso, a cura di Pietro G. Beltrami, Maria Grazia Capusso, Fabrizio Cigni, Sergio Vatteroni, Pise, Pacini, 2006, pp. 1595-1603. 
«Sur la date et l'auteur du remaniement du Roman de la Rose par Gui de Mori », dans Romania, t. 124 (2006), pp. 361-377.

Cet article a reçu le prix 2006 de l'AIEF et a été republié dans les Cahiers de l'Association internationale des études françaises, t. 59 (2007), pp. 361-381.

\section{Groupes de recherche}

« Histoire de la philologie romane », rattaché à la chaire de Littératures de la France médiévale, Collège de France.

«Translations médiévales: cinq siècles de traductions en français $\left(\mathrm{XI}^{\mathrm{e}}\right.$ $\mathrm{XV}^{\mathrm{e}}$ siècle) », projet coordonné par le Centre d'Études Supérieures de Civilisation Médiévale (CNRS — Université de Poitiers).

\section{ACTIVITÉS DU PROFESSEUR}

Publications

\section{Direction d'ouvrages collectifs}

Froissart dans sa forge. Colloque réuni à Paris, du 4 au 6 novembre 2004, par M. Michel Zink, Paris, Académie des inscriptions et belles-lettres - Collège de France, Diffusion de Boccard, 2006, 238 p. (contributions propres de Michel Zink : «Avant-propos », pp. 5-6 ; «Froissart dans sa forge », pp. 85-89 ; «Synthèse des communications », pp. 231-234).

L'homme face aux calamités naturelles dans l'Antiquité et au Moyen Âge, éd. Jacques Jouanna, Jean Leclant et Michel Zink, Cahiers de la villa « Kérylos », $\mathrm{n}^{\circ}$ 17, Paris, Diffusion de Boccard, 2006, XVIII-293 p.

\section{Articles}

«Étudier la littérature aujourd'hui », dans Univers du texte. Genèse, Fonction et Constellation, éd. Shoichi Sato, Granduate School of Letters, Nagoya University, 2006, pp. 197-206.

«Le Livre du roy Rambaux de Frise et du roy Brunor de Dampnemarche. Un exemplum du bon gouvernement», dans Remembrances et resveries. Hommage à Jean Batany, Orléans, Paradigme, 2006, pp. 335-343.

«Humaniste», dans Éthique de la philologie - Ethik der Philologie, éd. Ursula Bähler, Studien des Frankreich-Zentrums der Albert-Ludwigs-Universität Freiburg - Études du Centre français de l'Université Albert Ludwig de Fribourg, t. 14, Berliner Wissenschafts-Verlag, 2006, pp. 13-19.

«Une naissance de la poésie et de l'amour », préface à Le Moyen Âge flamboyant. Poésie et peinture, Paris, Diane de Selliers, 2006, pp. 13-31.

Préface à La légende du Graal dans les littératures européennes, sous la direction de Michel Stanesco, Paris, Le Livre de Poche, La Pochothèque, 2006, pp. 5-7. 
«Henri Pourrat, Le Mauvais Garçon », dans Voix croisées autour du Mauvais Garçon, Cahiers Henri Pourrat 21, Clermont-Ferrand, Bibliothèque communautaire et interuniversitaire, 2006, pp. 9-28.

"Quelques remarques sur Rerum vulgarium fragmenta 70 », dans Pétrarque et l'Europe, sous la direction de Carlo Ossola, Grenoble, Éditions Jérôme Millon, 2006, pp. 65-73.

«Les calamités et la conscience du poète au Moyen Âge », dans L'homme face aux calamités naturelles dans l'Antiquité et au Moyen Âge, éd. J. Jouanna, J. Leclant et M. Zink, Cahiers de la villa «Kérylos », n 17, Paris, Diffusion de Boccard, 2006, pp. 265-277.

« D’Apollonius de Tyr à Tristan. Roman volé, roman voilé », dans Los caminos del personaje en la narrativa medieval. Actas del colloquio internacional, Santiago de Compostela, 1-4 diciembre 2004, dir. Pilar Lorenzo Gradín, Florence, Edizioni del Galluzo, 2006 (Archivio romanzo 12, Pubblicazioni della scuola di dottorato europea in filologia romanza 2), pp. 41-59.

«Von den Elementen zur Landschaft», dans Landschaften im Mittelalter. Herausgegeben von Karl-Heinz Spiess, Stuttgart, Franz Steiner Verlag, 2006, pp. 199-206.

«La poésie comme récit», dans Cultural Performances in Medieval France. Essays in Honor of Nancy Freeman Regalado. Edited by Eglal Doss-Quinby, Roberta L. Krueger and E. Jane Burns, Cambridge, D.S. Brewer, 2007, pp. 3-13.

Préface à Bernard de Clairvaux. Sermons sur le Cantique. Tome V (Sermons 69-86). Introduction, traduction et notes de Paul Verdeyen et Raffaele Fassetta, Paris, Éditions du Cerf (Sources chrétiennes 511), 2007, pp. 21-24.

Comptes rendus dans Académie des inscriptions et belles-lettres: François Dolbeau, Sanctorum societas. Récits latins de sainteté (III ${ }^{e}$-XII ${ }^{e}$ siècles) (Comptes rendus des séances de l'année 2005, novembre-décembre, Paris, De Boccard, 2005 (parution 2007), pp. 1387-1393) ; Bulletin du Cange (Archivum Latinitatis Medii Aevi), 61 (2003) et 62 (2004), ibid., pp. 1393-1397.

\section{PARTiCIPATION À DES COLLOQUES}

3-4 octobre 2006, Université de Sienne. Colloque : «Tra Filologia e Comparatistica. Le riviste e la fondazione della Filologia romanza». Communication : «Romania. La naissance de la philologie romane en France ».

25 octobre 2006, Fondation Singer-Polignac. Colloque: «La musique au Moyen Âge ». Présidence du colloque. Exposé de conclusion et synthèse finale des communications.

$1^{\text {er }}$-4 novembre 2006, Université de Princeton. Colloque : «Poetry, Knowledge and Community in Late Medieval France». Conférence plénière de clôture : «Les razos et l'idée de la poésie». 
27 février- $1^{\text {er }}$ mars 2007, Université de Metz. Colloque : «Lettres et musique en Lorraine au XIII ${ }^{\mathrm{e}}$ siècle. Autour du Tournoi de Chauvency (Ms. Oxford Bodl. Douce 308)». Communication : «On connaît la chanson. Des échos familiers : chansons insérées et accent alsacien dans Le Tournoi de Chauvency».

14-16 mars 2007, Université de Bonn. Colloque: «Literaturgeschichte als Europäische Gründungs-mythos. L'Histoire littéraire comme mythe fondateur de l'identité européenne ». Conférence d'ouverture : «La poésie comme origine ».

20-21 avril 2007, Mount Holyoke College (South Hadley, Massachusetts) : «Music and Texts : The Middle Ages and Beyond. A Colloquium in Honor of Professor Margaret L. Switten». Communication: «La chanson volée. Arnaut Daniel, Anc ieu non l'aic, mas elha m'a (BdT 29, 2) et sa razo».

18-23 juin 2007, Fondation des Treilles (Tourtour, Alpes maritimes) : «Le Moyen Âge et la Renaissance au Collège de France». Organisation du colloque avec Pierre Toubert. Communication : «Les enjeux de la philologie médiévale à travers les leçons inaugurales du Collège de France ».

\section{CONFÉRENCES}

Université de Nagoya (Japon), première Superlecture du Institute for Advanced Research : «Une source de la poésie occidentale : les chansons des troubadours et leurs commentaires» (10 octobre 2006). — Université Harvard, Program of Medieval Studies : "The Razos of the Troubadours. An Idea of Poetry» (6 novembre 2006). - Paris, Cinéma «Le Champo»: «Perceval le Gallois d'Éric Rohmer» (20 mars 2007). 
